\title{
Influencing Factors and Strategies of the Flow of Academic Professionals in Colleges and Universities Based on Convolutional Neural Networks
}

\author{
LIli Zhao \\ Faculty of Public Administration, China University of Mining and Technology, Xuzhou 221000, Jiangsu, China \\ Correspondence should be addressed to LIli Zhao; lb18090056@cumt.edu.cn
}

Received 9 June 2021; Revised 12 July 2021; Accepted 13 August 2021; Published 23 August 2021

Academic Editor: Sang-Bing Tsai

Copyright (c) 2021 LIli Zhao. This is an open access article distributed under the Creative Commons Attribution License, which permits unrestricted use, distribution, and reproduction in any medium, provided the original work is properly cited.

\begin{abstract}
The influencing factors of academic professional mobility in colleges and universities are complex and diverse, and the intensity of each influencing factor has obvious characteristics of differentiation. However, scholars have few relevant studies based on the intensity of factors affecting the flow of academic professionals in colleges and universities. Therefore, this article aims to study and analyze the influence of different factors on the flow of academic professionals in different types of colleges and universities, which is of great significance for promoting the rational and orderly flow of academic professionals. This paper proposes a comprehensive application of multiple methods, such as questionnaire surveys and data statistics, based on ERG theory, based on the four types of universities and colleges: research-oriented, teaching research, teaching, and application. The influence intensity of the flow-influencing factors is comparatively studied. It clarified the differences in the influence of spiritual factors, economic factors, and social factors on the mobility of academic professionals in colleges and universities, established a mobility factor model, and put forward policy recommendations for colleges and universities to promote the rational and orderly mobility of academic professionals. A total of 2042 questionnaires on "Policy Improvement Factors Affecting the Flow of Academic Experts in Universities" were released, four academic experts of different levels were assigned to universities, and 1,561 were effectively searched. Among them, there were 336 research universities, 157 educational research universities, 404 educational universities, and 164 applied universities. The experimental results of this article show that the factors affecting the flow of academic professionals in universities include economic strength factor of 0.4945 , social strength factor of 0.5456 , and intellectual strength factor of 0.52 . Therefore, the factors affecting the mobility of university scholars can be used in strategic research.
\end{abstract}

\section{Introduction}

1.1. Background. A good talent training and development system is the foundation for the rapid progress of a country and nation, and education in this system is one of the main ways to develop and train talents. While developing, colleges and universities have also entered the stage of educational reform and have put forward high-level and high-level development requirements. They are also actively improving their own educational concepts, educational capital, educational models, and teaching facilities. In recent years, colleges and universities have continued to expand their student enrollment, so the demand for academic professionals in colleges and universities is increasing. As the main driving force for the development of higher education, university academic professionals have gradually become the main educational resource for competition among universities. The lack of talents and the growing demand for talents have further intensified the competition among academic professionals among universities. The reasonable flow of talents is beneficial and necessary for the development of the university, but the frequent flow of talents and the concentration effect of the flow of talents have an adverse effect on the development of the university. The frequent flow of academic professionals affects the stability of the development of education. This not only harms the development of the overall teaching level of colleges and universities, but also affects the self-development of high-level talents in colleges 
and universities. Therefore, how to reasonably regulate the flow of high-level talents in my country's universities is related to the prosperity and development of my country's higher education.

1.2. Significance. The operating quality of the academic labor market is of extreme importance to the government, universities, and scholars. For the government, the level of a country's academic labor force determines the level of higher education development and technological innovation in the era of knowledge economy. Under the combined effect of the globalization of higher education and the new public management, the international connections between universities have become closer and the competition between each other has become more intense. Scholar resources, as the core of the competition, have become the focus of priority competition among universities. The international flow of high-level human resources will determine the reordering of the status of the global technology center. For universities, as a nonprofit organization gathered by scholars, the maximization of prestige is its basic interest, and how to attract and retain talents is the center of personnel work. The type, location, and prestige of the initial academic position even determine the limit of future academic achievement. If the academic labor market cannot effectively allocate academic resources, it will lead to a mismatch between scholars and academic posts, resulting in a large loss of academic human capital.

1.3. Related Work. For a long time, the brain drain has been worrying. Zhou et al.'s research found that little is known about the brain drain in a country. China is a developing country that is not only experiencing the loss of overseas talents, but also experiencing domestic cross-regional and cross-sectoral flows. In this research, he studied the flow of high-level talents (HLT) based on the background of establishing world-class universities and disciplines (WCUD) in China and its dynamic mechanism and then discussed its potential impact. The results show that in the past four decades, eastern China has been a net inflow region, while the northeast and central and western regions have seen net outflows. The eastern region shows more internal regional mobility. In addition, a large amount of HLT flows from research institutions to universities (CU). Regional socioeconomic gaps, imperfect systems, and poor management are the main reasons for the movement of high-level personnel [1]. However, due to the uncertainty of the experimental process, there are still gaps in the experimental results. The dispersion of talent within the United States is not uniform. Joseph et al.'s research found that there is sufficient statistical evidence that there is an interstate brain drain phenomenon within the country. They first studied this by determining whether the country can be divided into four categories of talents. To this end, Joseph et al. observed the relative pull or driving force of talents and observed relative to which states tend to retain or lose their native talents and which states tend to attract a large or small number of immigrant students seeking education results outside their own state. Once they have completed this classification, Joseph et al. will try to see if there is a set of randomly selected independent attributes or topics in these groups that are statistically important to support these classifications [2]. However, their experimental process is not closed, so there is a certain deviation in the experimental results. Regional competitiveness depends on the power of science and technology; the development of politics, economy, and culture affects the demand for talents and factors that affect the flow of science and technology personnel. Therefore, there is an urgent need to solve the problem of how to create a good environment and attract talents to achieve effective management of them. Zhao et al. used factor analysis, cluster analysis, multidimensional online analysis, and regression analysis to explore the relationship between regional environments, provide guidance for the construction of regional environments, and promote the rational flow of scientific and technological talents. They found that different regions have different factors. The expected elements of scientific and technological personnel in eastern provinces are the level of economic development and income, while the living environment and cultural environment are the main expected elements in the central and western regions [3]. However, due to the unclosed nature of the experiment, there are still some discrepancies in the experimental data.

1.4. Innovation. The innovation of this research lies in the following: (1) it puts forward the influencing factors of academic professionals in colleges and universities, analyzes the intensity of the factors affecting the mobility of academic professionals in four different colleges and universities, and establishes a mobility factor model and (2) it puts forward the viewpoint that the factors affecting the mobility of academic professionals in colleges and universities are divided into "three forces," such as spiritual factors, economic factors, and social forces.

\section{Convolutional Neural Networks}

A typical convolutional neural network consists of a series of processes. The function of the subsampling layer is to sample the feature map output by the convolutional layer. The sampling layer is sampled by scanning the step size of the sampling area instead of continuous sampling. Among them, the coacervate and concentration layers are the first step. The adhesive layer units are organized into feature maps. In the feature map, each unit transfers a set of weights, which are linked to the feature-level part of the upper level. The exclusion and this local weighted sum are transferred to a nonlinear function (usually called an activation function) $[4,5]$. The basic structure of the convolutional neural network is shown in Figure 1.

2.1. Convolutional Layer. The role of the convolutional layer is essentially to extract local features, and then the role of the pooling layer is to combine semantically similar features [6]. Usually, the pooling layer calculates the maximum value of 


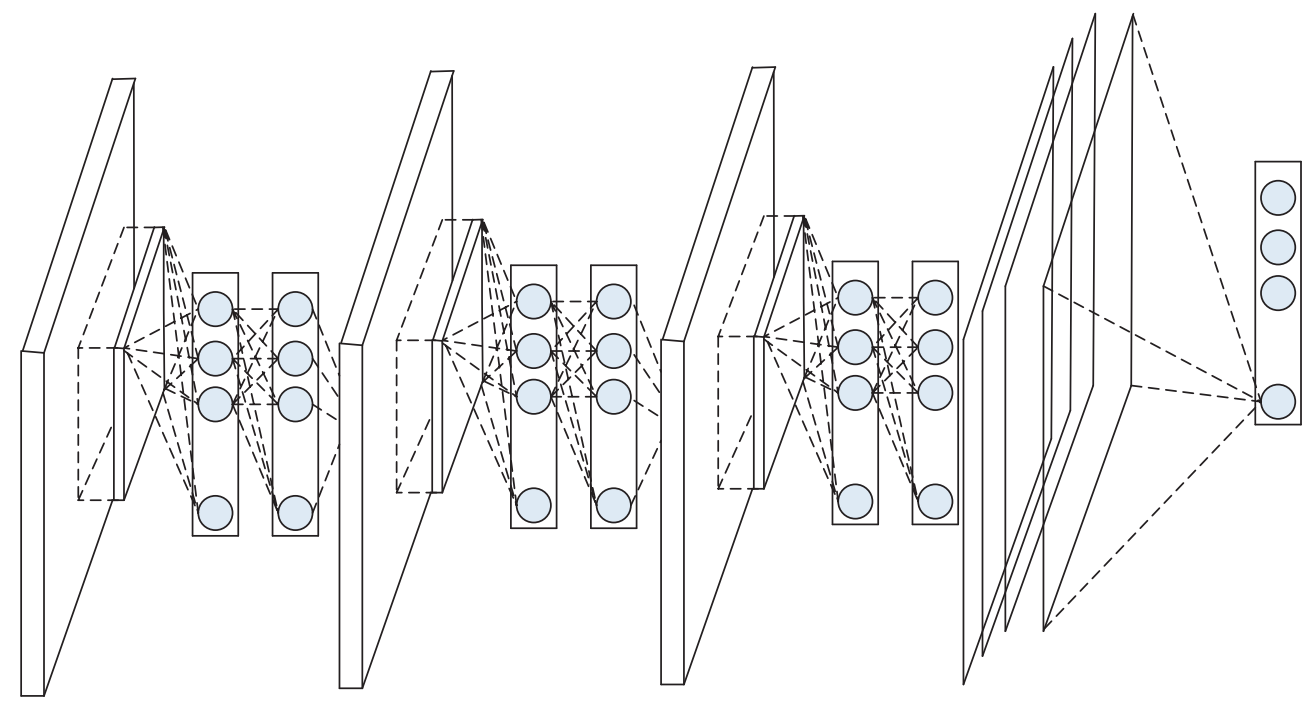

Figure 1: Basic structure of the convolutional neural network.

the local block in the feature map, and the adjacent concentrated neuron reads data from the small block by moving rows or columns. This is to reduce the dimensionality and immutability of the data $[7,8]$ :

$$
O=\left(\sum \sum I(i, j)^{P} \times G(i, j)^{1 / P}\right) .
$$

Here, $I$ represent the input feature map, $G$ represents the Gaussian kernel, 0 represents the output feature map, and the value of $P$ is selected from 1 to $\infty$. For $P-1$, the subsampling layer performs mean sampling and each subregion will be calculated. The mean value in is used as the subsampling result [9]; when $P \longrightarrow \infty$, the subsampling layer performs maximum sampling, and the maximum value in each subregion will be selected as the subsampling result. The sampling diagram is shown in Figure 2.

Suppose there are $m$ labeled training samples $\left\{\left(x_{1}, y_{1}\right)\right.$, $\left.\left(x_{2}, y_{2}\right), \ldots,\left(x_{m}, y_{m}\right)\right\}$, in the training set, where the input feature $x^{(i)} \in R^{n+1}$, because it is used for binary classification in logistic, so the category label $y^{(i)} \in\{0,1\}[10,11]$. The hypothesis function (hypothesis function) is as follows:

$$
h_{\theta}(x)=\frac{1}{1+\exp \left(-\theta^{T} x\right)} .
$$

We need to minimize the following cost function by training model parameters:

$$
J(\theta)=-\frac{1}{m}\left[\sum_{i=1}^{m} y^{(i)} \log h_{\theta}\left(x^{(i)}\right)+\left(1-y^{(i)}\right) \log \left(1-h_{\theta}\left(x^{(i)}\right)\right)\right] .
$$

In the given test input data $x$, we need to use a hypothesis function to estimate the probability value of each category of $j(j=1,2, \ldots, k) p(y=j \mid x)$, that is, When $j=1,2, \ldots, k$, then there is $p(y=j \mid x)$. When appearing as input, the probability of each classification result appears [12]. So, our hypothesis function needs to output a $k$-dimensional vector (the sum of each dimensional element component is 1), and each dimensional element component represents the probability that the input $x$ belongs to this category $[13,14]$. This hypothetical function has the following form:

$$
h_{\theta}\left(x_{i}\right)=\left[\begin{array}{c}
p\left(y_{i}=1 \mid x_{i} ; \theta\right) \\
p\left(y_{i}=2 \mid x_{i} ; \theta\right) \\
\cdots \\
p\left(y_{i}=k \mid x_{i} ; \theta\right)
\end{array}\right]=\frac{1}{\sum_{j=1}^{k} e^{\theta_{j}^{T} x_{i}}}\left[\begin{array}{c}
e^{\theta_{1}^{T} x_{i}} \\
e^{\theta_{2}^{T} x_{i}} \\
\cdots \\
e^{\theta_{k}^{T} x_{i}}
\end{array}\right] .
$$

Here, $h_{\theta}\left(x_{i}\right)$ represents the parameters of the model, and the fraction $1 / \sum_{j=1}^{k} e^{\theta_{j}^{T} x_{i}}$ is used for normalization operation to ensure that the sum of the elements of each dimension of the vector is 1 [15].

The cost function of the Softmax classifier is as follows:

$$
J(\theta)=-\frac{1}{m}\left[\sum_{i=1}^{m} \sum_{j=1}^{k} 1\left\{y_{i}=j\right\} y^{(i)} \log \frac{e^{\theta_{j}^{T} x_{i}}}{\sum_{j=1}^{k} e^{\theta_{j}^{T} x_{i}}}\right] .
$$

Formula (5) can be used as a further extension of the logistic regression cost function $[16,17]$. The logistic regression cost function can be changed to

$$
\begin{aligned}
J(\theta)= & -\frac{1}{m}\left[\sum_{i=1}^{m} y^{(i)} \log h_{\theta}\left(x^{(i)}\right)+\left(1-y^{(i)}\right) \log \right. \\
& \left.\left(1-h_{\theta}\left(x^{(i)}\right)\right)\right] \\
= & -\frac{1}{m}\left[\sum_{i=1}^{m} \sum_{j=1}^{k} 1\left\{y_{i}=j\right\} \log p\left(y^{(i)}=j \mid x^{(i)} ; \theta\right)\right], \\
p\left(y^{(i)}=\right. & \left.j \mid x^{(i)} ; \theta\right)=\frac{e^{\theta_{l}^{T} x_{i}}}{\sum_{l=1}^{k} e^{\theta_{l}^{T} x_{i}}}
\end{aligned}
$$

Regarding the minimization of $J(\theta)$, there is no closedform solution [18]. We usually use iterative optimization algorithms, such as gradient descent algorithm. Through 


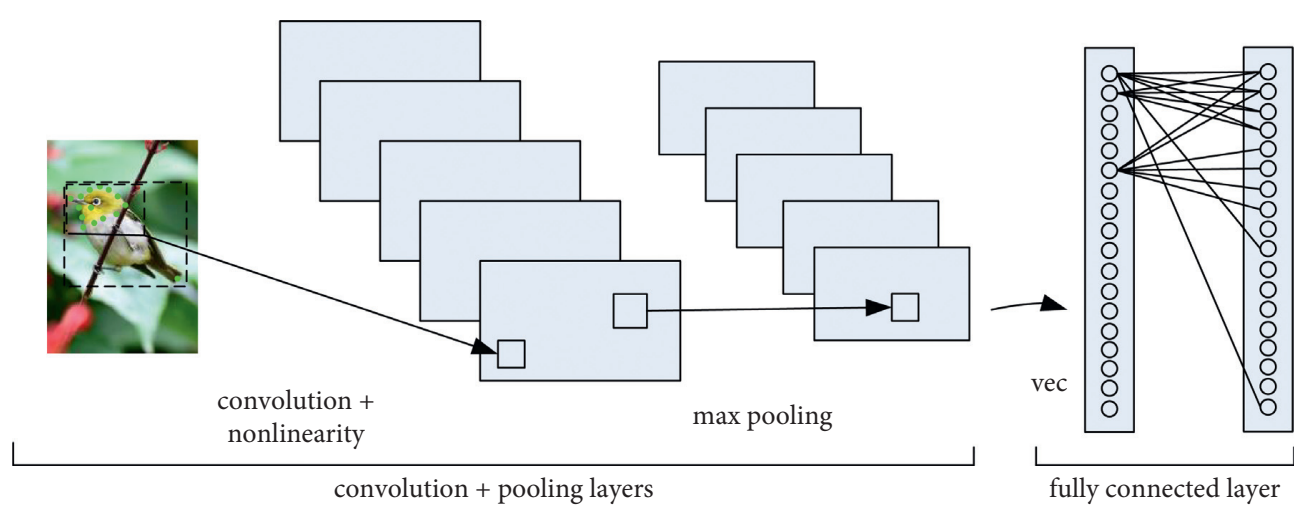

FIgURE 2: Schematic diagram of sampling.

derivative calculation, the following gradient formula is obtained:

$$
\left.\nabla_{\theta_{j}} J(\theta)=-\frac{1}{m} \sum_{i=1}^{m}\left[x_{i}\left(1\left\{y_{i}=j\right\}\right)\right]-p\left(y_{i}=j \mid x_{i} ; \theta\right)\right] .
$$

2.2. Training of Convolutional Neural Networks. For convolutional neural networks, generally supervised methods are directly used for training, and supervised methods usually use gradient-based methods. The batch stochastic gradient descent method is usually used $[19,20]$. The error function formula for sample $n$ is as follows:

$$
J(W, b ; x, y)=\frac{1}{2} \sum_{k=1}^{c}\left(t_{k}-y_{k}\right)^{2}=\frac{1}{2}\|t-y\|^{2} .
$$

Here, $W$ and $b$, respectively, represent the weight and bias of the neural network and $x$ and $y$, respectively, represent the training sample and its corresponding label. $t_{k}$ represents the $k$-th dimension component of the predicted value of the sample $x, y_{k}$ represents the $k$-th dimension component of the label of the training sample $x$, and $t$ represents the predicted value corresponding to the training sample $x$.

Assuming that $\delta^{(l+1)}$ is the error term of the $l+1$ layer calculated according to the above formula, its weight and bias parameters are $W$ and $b$, respectively. If the $l+1$ layer is completely connected to the $l+1$ layer, the error term of the $l$ layer is calculated as follows:

$$
\delta^{(l)}=\left(\left(W^{(l)}\right)^{T} \delta^{(l+1)}\right) \cdot f^{\prime}\left(z^{(l)}\right)
$$

The corresponding gradient calculation formula is as follows:

$$
\begin{aligned}
& \nabla_{W^{(l)}} J(W, b ; x, y)=\delta^{(l+1)}\left(a^{(l)}\right)^{T}, \\
& \nabla_{W^{(l)}} J(W, b ; x, y)=\delta^{(l+1)} .
\end{aligned}
$$

If the first layer is the feature extraction stage, that is, the convolutional layer and the subsampling layer, the error term of the first layer is calculated by the following formula:

$$
\delta_{k}^{(l)}=\operatorname{upsample}\left(\left(W_{k}^{(l)}\right)^{T} \delta_{k}^{(l)}\right) \cdot f^{\prime}\left(z_{k}^{(l)}\right) .
$$

Here, $k$ represents the $k$-th convolution kernel, and the upsample $(\cdot)$ operation transfers the error $\delta_{k}^{(l+1)}$ calculated by the latter layer to the previous layer through the subsampling layer, that is, the convolutional layer [21]. For example, if we use mean sampling, upsample(.) will simply evenly distribute the error to the subregion where subsampling was previously performed. If the maximum sampling is used, then the subsampling operation will be performed during the previous forward propagation. The position selected as the sampling value will get all the errors, and the other positions will be 0 .

Finally, when calculating the gradient, we need to flip the convolution kernel as in the convolution operation. The calculation formula is as follows:

$$
\begin{aligned}
\nabla_{W_{k}^{(l)}} J(W, b ; x, y) & =\sum_{i=1}^{m}\left(a_{i}^{(l)}\right) * \operatorname{rot} 90\left(\delta_{k}^{(l+1)}, 2\right), \\
\nabla_{b_{k}^{(l)}} J(W, b ; x, y) & =\sum_{a, b}\left(\delta_{k}^{(l+1)}\right)_{a, b} .
\end{aligned}
$$

Here, $f^{\prime}(\cdot)$ is the steering function of the activation function $f(\cdot)$, the activation (output value) of layer $a^{(l)}$ and $l$, and $a^{(l)}$ is the input image. $a_{i}^{(l)} * \delta_{k}^{(l+1)}$ represents $k$ convolution kernels of $i$ input and error items on the $l$ layer for convolution operation [22, 23].

When the backpropagation ends, the gradient descent method is used to update the weights, and the update formula is as follows:

$$
\theta=\theta-\partial \nabla_{\theta} J(\theta ; x, y)
$$

Here, $\theta$ is the weight and bias parameters that need to be learned and $\partial$ is the learning rate.

\section{The Current Situation and Influencing Factors of the Flow of Academic Professionals in Colleges and Universities}

Scholars have done countless researches on the factors of university teacher mobility, but few have done research on the intensity of mobility factors. In order to carry out in- 
depth research, we divide the factors affecting the flow of college teachers into "economic factors," "social factors," and "mental factors" and use the difference in intensity to build a model of teacher mobility factors [24]. At the same time, due to differences in university positioning and development strategies, different types of colleges and universities have different factors affecting the flow of teachers. Therefore, colleges and universities are divided into four types: research-oriented universities, teaching and research universities, teaching universities, and applied universities, and starting research is necessary. In order to study the difference in the degree of influence of different factors on the flow of academic professionals in colleges and universities, we issued the "College Academic Professionals" to four different levels of college academic professionals in research-oriented universities, teaching and research universities, teaching universities, and applied universities. A total of 2,042 questionnaires were issued in the "Flow Influencing Factors and Policy Improvement," and 1,561 were effectively recovered. Among them, 336 are research-oriented universities, 157 are teaching and research universities, 404 are teaching universities, and 164 are applied universities. The questionnaire involved many issues such as the basic situation of mobile academic professionals, the structure of their academic background, the influencing factors of the flow, the flow intention, and the flow policy recommendations. Regarding the factors affecting the flow of academic professionals in colleges and universities, we conducted a survey on the influence of 30 factors, such as the legal environment, talent policy, and social climate. The influence of each factor is divided into "very important," "relatively important," and "five options are available: "general," "not so important," and "not important."

3.1. Comparison Based on the Intensity of Influencing Factors. In order to compare the intensity of the factors affecting the mobility of academic professionals in colleges and universities, we added weights to the intensities of the influence of each factor in four different types of colleges and universities and performed a summation. Assuming that the weight of "high intensity" is 3 , and the weight of "medium intensity" is 2 , and the weight of "low intensity" is 1 , then the total intensity of the income status = research-oriented mediumintensity $2+$ teaching-research high-intensity $3+$ teaching high-intensity $3+$ applied high-intensity $3=$ total intensity 11, as shown in Tables 1-3.

It can be seen from Tables 1-3 that self-development is the most important factor among the 15 factors affecting academic mobility of universities. The income system is more concentrated. Influencing factors are the awareness of academic professional team structure, vocational training, self-vision, and organizational vision. Housing conditions, early childhood education safety, welfare system, school level type, and academic performance will affect low-intensity factors. Finally, medical safety conditions, relevant laws and regulations, social mobility, and organizational culture are the least influential factors.
TABLE 1: Intensity of factors affecting the mobility of academic professionals in colleges and universities.

\begin{tabular}{lcc}
\hline College category & Total strength \\
\hline & Income status & 11 \\
& Housing conditions & 9 \\
Economic factor & Medical insurance conditions & 8 \\
& Children's education guarantee & 9 \\
& Welfare system & 9 \\
\hline
\end{tabular}

TABLE 2: Total intensities of influencing factors in the mobility of academic professionals in colleges and universities.

\begin{tabular}{lcc}
\hline & Relevant laws and regulations & 7 \\
Social factors & Social mobility & 6 \\
& School level type & 9 \\
& Organizational culture & 6 \\
& Faculty structure & 10 \\
\hline
\end{tabular}

TABLE 3: Intensity of factors influencing the mobility of academic professionals in colleges and universities.

\begin{tabular}{lcc}
\hline & Talent incentive system & 11 \\
& Personal development space & 12 \\
Mental power & Professional training opportunities & 10 \\
factor & Academic achievement & 9 \\
& Self-goal and organizational vision & 10 \\
& recognition & \\
\hline
\end{tabular}

3.2. Economic Factors. Through the study of employee behavior, it is found that employee behavior is usually affected by economic factors, and the pursuit of economic benefits is maximized. Without exception, the mobility of academic professionals will also consider the impact of economic factors. The economic factors referred to in this article are broad concepts [25]. In addition to the income status of academic professionals, they also include housing conditions, children's education security, medical security, and welfare system.

3.2.1. Income Status. Income status is remuneration paid in currency or other forms. The income of academic professionals in my country's universities includes wages and allowances and subsidies. Among them, wages are composed of basic salary, job allowances, and special job allowances stipulated by the state; allowances and subsidies are composed of job allowances, overwork subsidies, reform subsidies, holiday condolences, and other items. Among them, Figure 3 shows the intensity of the influence of "wage income" on academic professionals in different types of institutions [26].

It can be seen from Figure 3 that except for academic professionals in research-led universities who believe that "wage income" is a "medium-intensity influencing factor" for the mobility of academic professionals, academic professionals in the other three colleges all believe that "income status" affects academic careers in universities and "highintensity factor" of the flow of people. This is because, first of all, research-led university scholars have higher academic 


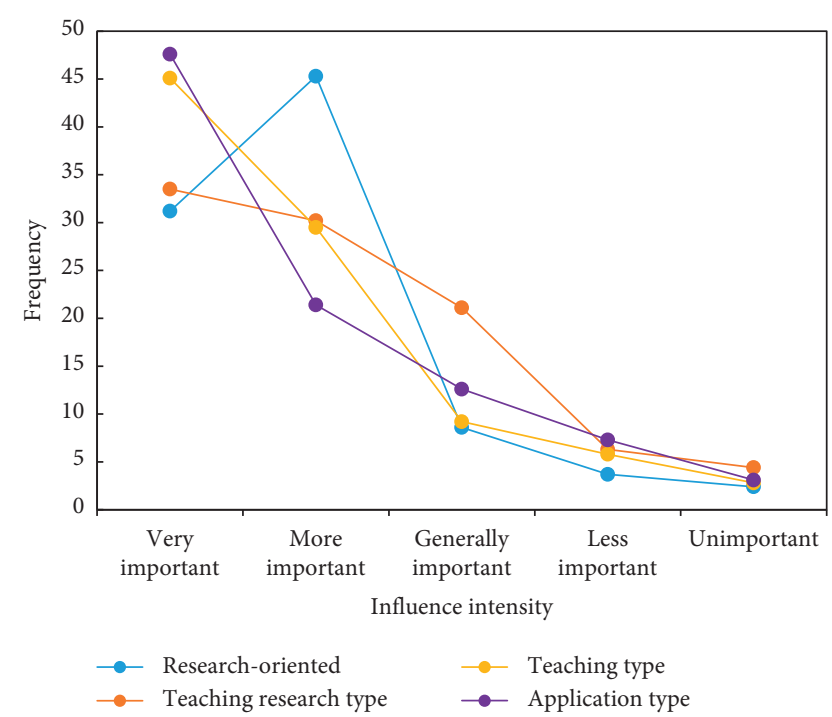

FIGURE 3: Intensity of the influence of "wage income" on academic professionals in different types of colleges and universities.

qualifications, higher academic abilities, and stronger mobility, so they pay more attention to meeting advanced needs, such as psychological needs. Regarding "salary income," other economic benefits are relatively good, and the degree of attention to "salary income" is lower than that of academic professionals in other types of universities.

3.2.2. Housing Conditions. For colleges and universities, raising funds to build houses has become an important way to solve the housing problem of academic professionals. In addition to raising funds to build houses, dormitories, turnover houses, and apartments are also other ways to solve the housing of academic professionals. However, colleges and universities will introduce new academic professions and exodus academic professions every year to solve the housing problem and ensure the quality of life, which becomes the top priority. In recent years, as housing prices across the country have doubled, the number of transitional houses in schools has been insufficient, academic professionals have continued to increase, and the waiting line has increased year by year. University academic professionals have been hindered from buying houses, and academic professionals with low income and no accumulation are in urgent need. Universities solve the housing problem. Colleges and universities usually provide one-time housing subsidies or adopt the "dual system" model of "monetary subsidies plus physical placement" to attract academic professionals to inflow. Figure 4 shows the intensity of the influence of "housing conditions" on academic professionals in different types of colleges and universities.

It can be seen from Figure 4 that academic professionals in applied universities believe that "housing conditions" are a "high-intensity influencing factor" for the mobility of academic professionals in colleges and universities, while academic professionals in the other three types of colleges all believe that "housing conditions" are the mobility of academic professionals in colleges and universities. This

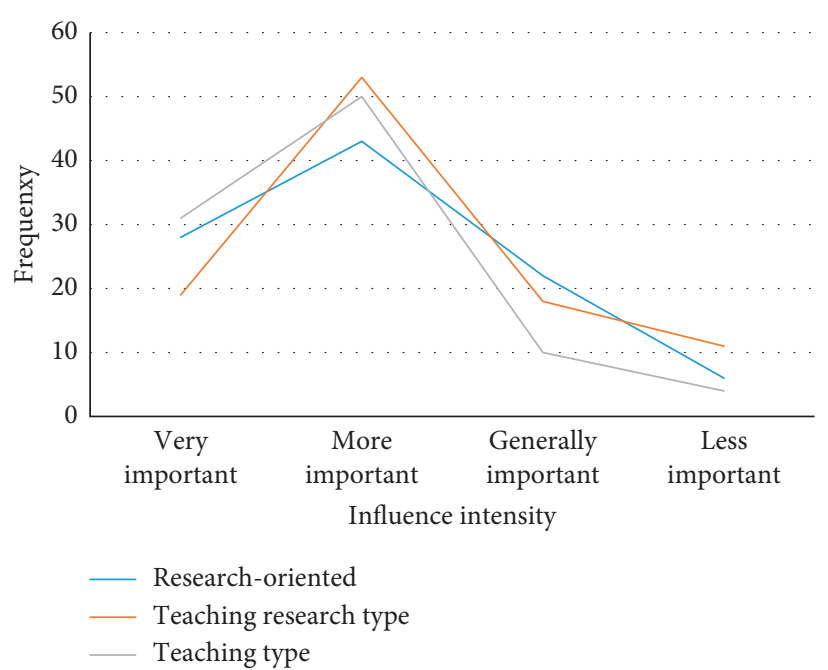

FIGURE 4: Intensity of the influence of "housing conditions" on academic professionals in different types of colleges and universities.

may be because academic professionals in research-led, teaching-research, and teaching universities have higher income levels and housing purchase welfare systems. Most academic professionals in such universities can buy their own houses without living in them. Regarding housing provided by the school, however, for academic professionals in applied universities, relatively low wages and welfare systems make housing conditions still a high-intensity influencing factor.

3.2.3. Children's Education Guarantee. The children of academic professionals have unique advantages in education. A large number of studies have proved that the academic performance of children of academic professionals is better than the children of parents of other occupations, and the success rate of children of academic professionals is higher. However, academic professionals will encounter a series of problems such as high expectations leading to lower eyes when educating children, stereotyped thinking leads to dislocation of roles, and busy work leads to negligence of communication. Therefore, the education of children of academic professionals often requires more attention. Figure 5 shows the intensity of the impact of children's education security on academic professionals in different types of colleges and universities.

It can be seen from Figure 5 that except for academic professionals in teaching and research universities who believe that "children's education security" is a "high-intensity influencing factor" that affects the mobility of academic professionals in colleges and universities, the other three college academic professionals believe that "children's education security" is the "medium-intensity influencing factor" of the mobility of academic professionals. This may be based on the fact that most academic professionals in teaching and research universities must not only take into account scientific research work but also do a good job in teaching. Taking multiple positions allows them to realize the importance of 


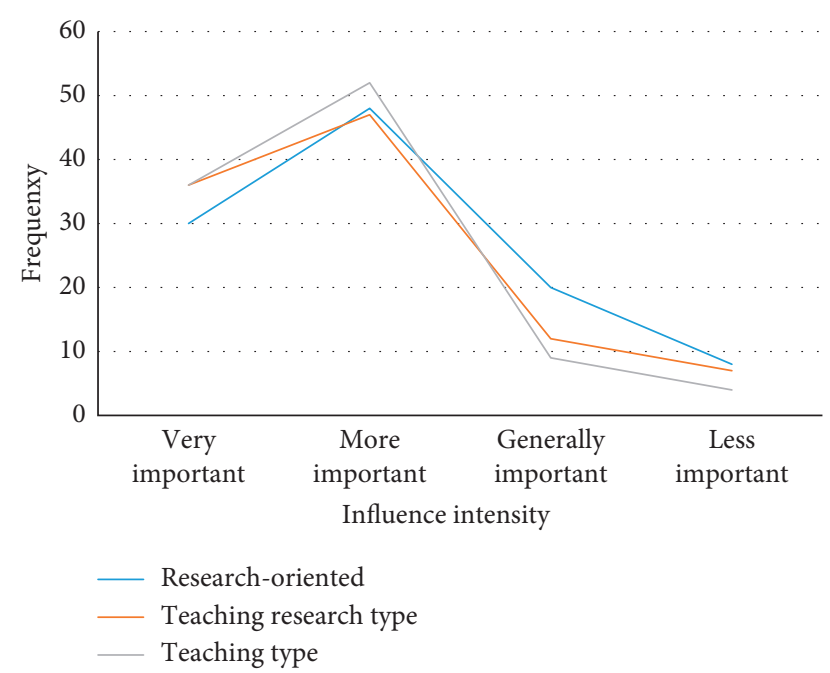

FIGURE 5: Intensity of the impact of children's education security on academic professionals in different types of colleges and universities.

cultivating multiple abilities, and primary and secondary schools, especially the elementary level; it is an important stage of multifield and all-round ability training, so they pay more attention to the education of their children.

3.3. Analysis of Social Factors. Mayo's "social man" hypothesis puts forward that social needs are the basic needs of "social man." People gain a sense of identity in social interactions. Compared with formal organizations, informal organizations have a greater impact on human behavior. The same is true for academic professionals in colleges and universities, whose mobility behavior is usually affected by social factors. According to Mayo's hypothesis, academic professionals in colleges and universities, social people, social needs, and gaining a sense of identity are the main influencing factors of social forces.

3.3.1. Relevant Laws and Regulations. The lack of perfect systems and norms for the flow of academic professionals in colleges and universities has led to frequent and disorderly flow of academic professionals. At the macrolevel, it is mainly manifested as the role misalignment between national regulation and the independent development of academic professionals. At the microlevel, it is manifested as the low efficiency of university management. Figure 6 shows the intensity of the influence of "related laws and regulations" on academic professionals in different types of colleges and universities. The changes in university teacher mobility policies are mainly manifested in the changes in mobility concepts, mobility mechanisms, and willingness to flow. The overall presentation has evolved from a mandatory government-led university faculty mobility policy to a "government guidance + market participation" university faculty mobility policy."

It can be seen from Figure 6 that except for academic professionals in teaching and research universities that "relevant laws and regulations" are the "low-intensity influencing factors" for the mobility of academic professionals in colleges and universities, academic professionals in the other three colleges all believe that "relevant laws and regulations" are the "medium-intensity influencing factor" of the mobility of academic professionals. This may be based on the fact that academic professionals in teaching and research universities hold multiple positions, and laws and regulations are less restrictive than those of the other three types of academic professionals.

3.3.2. School Level Type. For academic professionals, the university level is not only a consideration of external prestige, but also a concern for university resources. Often, the higher the level of the institution, the more the resources they get. In the context of "double first-class," "first-class universities" and "first-class disciplines" are more attractive to academic professionals; on the contrary, lower-level colleges and universities have fewer resources, attracting academic professionals to flow into the comparison difficult. Figure 7 shows the intensity of the impact of school level types on academic professionals in different types of institutions.

It can be seen from Figure 7 that except for academic professionals in research-led universities who believe that "school level type" is a "high-intensity influence factor" for the flow of academic professionals in colleges and universities, academic professionals in the other three colleges all believe that "school level type" is "mid-strength influencing factors." This may be because the resources required by the research of academic professionals in research-leading universities are directly linked to the level of the school, so they will choose the corresponding university based on their scientific research capabilities.

\section{Influencing Factors and Strategy Research on the Flow of Academic Professionals in Colleges and Universities}

In order to analyze the characteristics of the factors affecting the mobility of academic professionals in research-oriented, teaching-research, teaching and application-oriented universities, we divided 15 factors influencing the mobility of academic professionals in colleges and universities into "economic factors" and "social factors." The three types of "mental factors" compare the influence of the "three factors" on the flow of academic professionals in four different types of colleges and universities.

4.1. Intensities of Factors Affecting the Mobility of Academic Professionals in Different Types of Colleges and Universities. In order to compare the influence intensity of "economic factors," "social factors," and "mental factors" based on the types of institutions, we have drawn the following summary table based on Tables 1-3. Assuming that the sum of the three strength distributions for each force is $100 \%$, the distribution ratio is calculated according to the number of factors that are distributed among the "three force factors." 


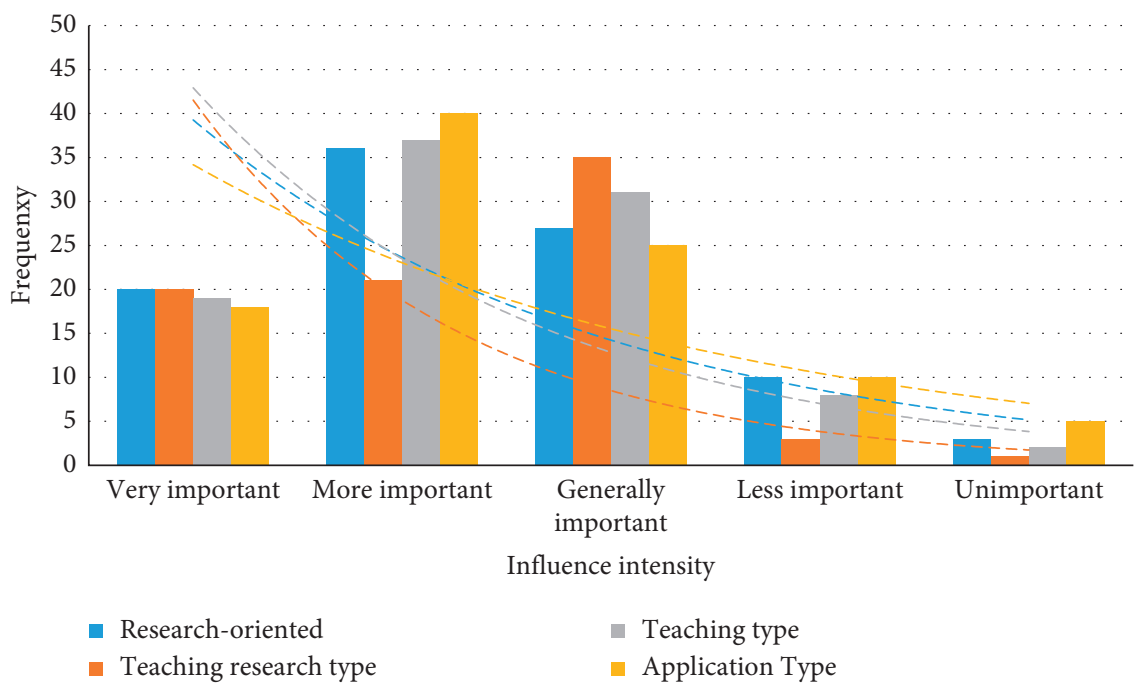

FIGURE 6: "Relevant laws and regulations" influence intensity of academic professionals in different types of colleges and universities.

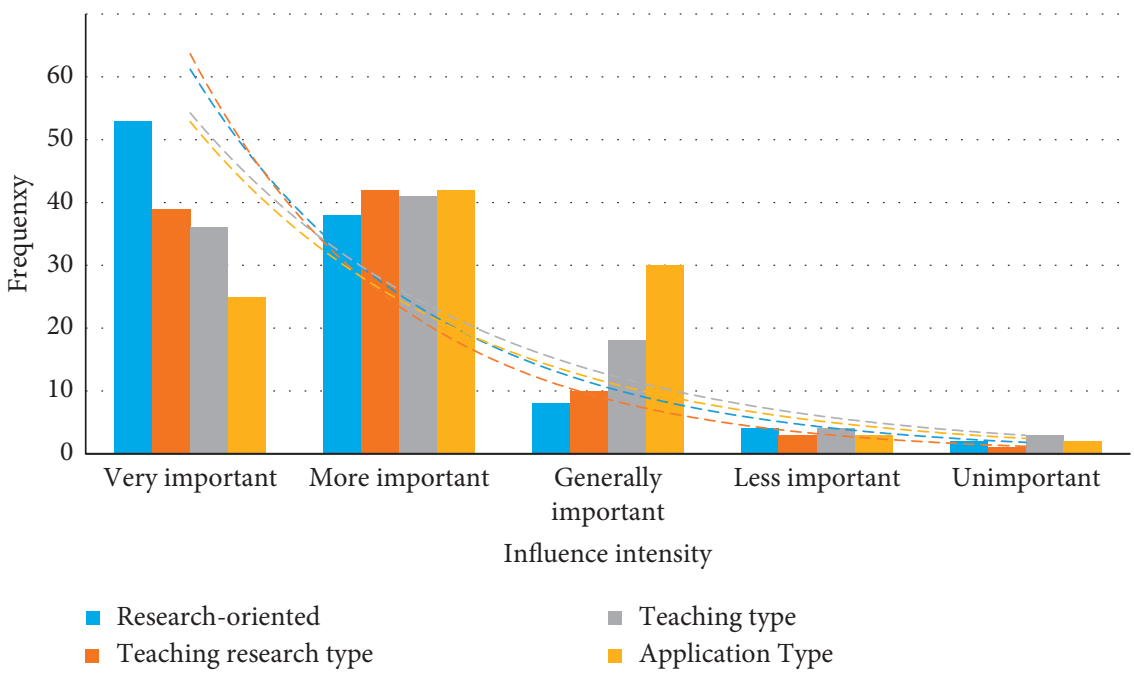

Figure 7: Intensity of the impact of school level types on academic professionals in different types of institutions.

Based on the type of colleges and universities, the impact of the "three forces" is shown in Table 4.

According to Table 4, we find that "economic power factors," "social power factors," and "mental power factors" have different influences on the mobility of academic professionals in four different types of colleges and universities. The factors of economic power and mental power are all located in the middle and high intensity, and the distribution of social power factors is different in the high intensity. In order to make the results easier to observe, we weighted and summed the three forces of economic power, social power, and mental power, respectively. The weight of low strength is 1 , the weight of medium strength is 2 , and the weight of high strength is 3 . Figure 8 is calculated.

It can be seen from Figure 8 that for the flow of academic careers in research-led universities, the influence of mental factors is the highest, and the influence of social factors and economic factors is at the same level; for academic careers in teaching-research, teaching, and application-oriented universities, in terms of mobility, the influence of mental factors is the highest, followed by economic factors, and the influence of social factors is relatively low.

4.2. Adjusting the Mobility Policy of Academic Professionals Based on the Difference in the Strength of the "Three Forces". The inequality in the regional distribution of ordinary universities, especially the concept of "double first-rate," has exacerbated the "differentiation between the rich and the poor" between the regions, and the management resources of universities are relatively large. This resource gap is evident in the different resources of academic professionals. This resource gap is prominently manifested in the difference in resources of academic professionals. As the core competitiveness of colleges and universities, academic professionals have relatively high differences between their academic qualifications, professional titles, and other "selfinducing factors" and graduate colleges and other 


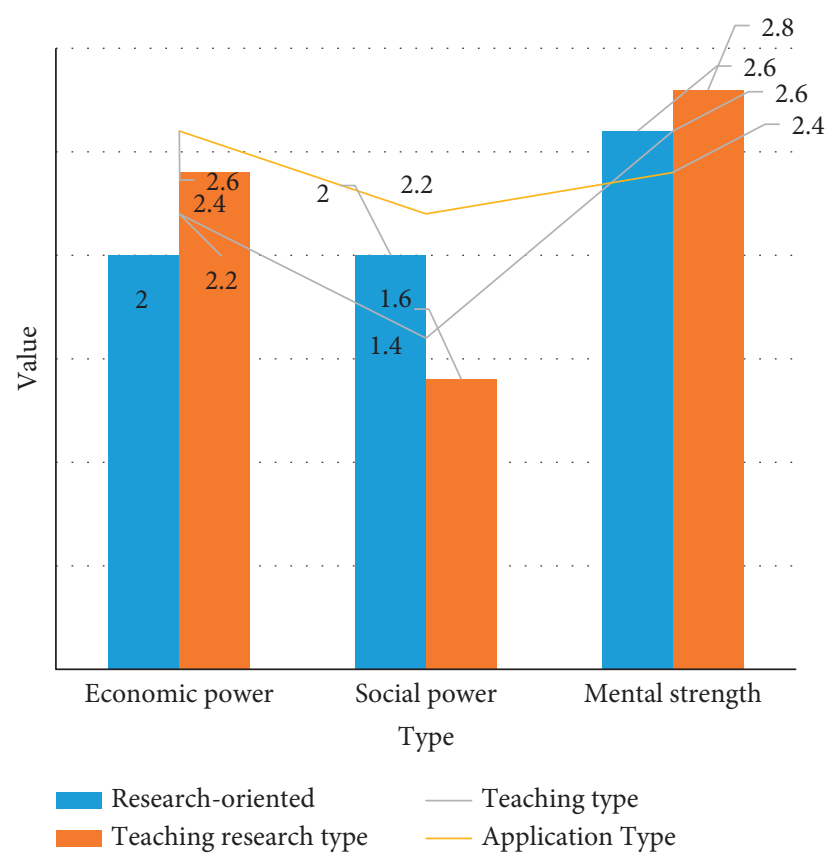

FIGURE 8: Radar chart of the influence intensity of the "three forces" based on the type of institutions.

TABLE 4: Intensity of the influence of the "three forces" on the basis of the type of institution.

\begin{tabular}{|c|c|c|c|c|c|c|c|c|c|}
\hline & \multicolumn{3}{|c|}{ Economic power } & \multicolumn{3}{|c|}{ Influence } & \multicolumn{3}{|c|}{ Mental strength } \\
\hline & Low & Medium (\%) & High (\%) & Low (\%) & Medium (\%) & High (\%) & Low & Medium (\%) & High (\%) \\
\hline Research-oriented & & 100 & & 20 & 60 & 20 & & 40 & 60 \\
\hline Teaching research type & & 60 & 40 & 40 & 20 & 20 & & 20 & 80 \\
\hline Teaching type & & 80 & 20 & 40 & 60 & & & 40 & 60 \\
\hline Application type & & 40 & 60 & & 80 & 20 & & 60 & 40 \\
\hline
\end{tabular}

"belonging factors." These differences make academic professionals different in their work focus. Academic professionals in leading universities have an advantage in scientific research, and academic professionals in teaching universities have an advantage in teaching activities. However, it is impossible for any university to focus solely on "research" or "teaching." Both must be considered. It requires both academic professionals who are good at scientific research and academic professionals who are good at teaching. Therefore, it is necessary for colleges and universities to attract the influx of academic professionals who need typology from other types of institutions. Because most indicators are involved in this study, we will analyze the reliability of each dimension separately and then analyze the reliability of the overall scale. The results of the analysis are shown in Table 5.

From Table 5, we can see that the alpha coefficient of 9 indicators in personal attributes is 0.752 , the alpha coefficient of 16 indicators in organizational attributes is 0.854 , the alpha coefficient of 4 indicators in social attributes is 0.731 , and the overall alpha coefficient of the scale is 0.916 , and the alpha coefficients of the three dimensions are all higher than 0.7, and all are in the range of 0.7-0.98. Therefore, the measurement results of the scale in these three dimensions have high credibility, and the overall credibility of the scale it is also in the range of high confidence.
4.3. Grasping the Law of the Strength of the "Three Forces" and Standardizing the Mobility of Academic Professionals. In the field of practice, it is necessary to actively explore the flow of academic professionals in colleges and universities. On the one hand, colleges and universities do not understand the intensity of the factors affecting the flow of academic professionals and spontaneously raise the level of some factors, which not only failed to promote the flow of academic professionals but also caused a waste of college resources. On the other hand, colleges and universities cannot provide the conditions for academic professionals in colleges and universities, and the flow of academic professionals is hindered or causes disorderly flow of academic professionals. Therefore, colleges and universities have a certain degree of blindness when using resources to promote the flow of academic professionals. After conducting a horizontal analysis, we have made a longitudinal comparative analysis of the correlation between the influencing factors in the three dimensions of economic power, social power, and spiritual power and the flow of academic professionals. The results of the analysis are shown in Table 6.

The mean value of Pearson's correlation in Table 6 is obtained by calculating the mean value of Pearson's correlation of all influencing factors in each dimension. In order to regulate the mobility of academic professionals, colleges 
TABLE 5: Reliability analysis.

\begin{tabular}{lcc}
\hline & Cronbach's alpha & $N$ of items \\
\hline Economic factors & 0.752 & 9 \\
Social factors & 0.854 & 16 \\
Spiritual factors & 0.731 & 4 \\
Overall scale & 0.916 & 29 \\
\hline
\end{tabular}

TABLE 6: Summary of correlation analysis.

\begin{tabular}{lccc}
\hline $\begin{array}{l}\text { Relevance to teacher } \\
\text { mobility }\end{array}$ & $\begin{array}{c}\text { Economic } \\
\text { factors }\end{array}$ & $\begin{array}{c}\text { Social } \\
\text { factors }\end{array}$ & $\begin{array}{c}\text { Spiritual } \\
\text { factors }\end{array}$ \\
\hline $\begin{array}{l}\text { Pearson correlation } \\
\text { mean }\end{array}$ & 0.4945 & 0.5456 & 0.52 \\
\hline
\end{tabular}

and universities should understand the intensity of the factors affecting the mobility of academic professionals in various types of colleges and universities, formulate strategies for the introduction of academic professionals in colleges and universities, and promote the mobility of academic professionals in a planned and purposeful manner. Whether it is a research-led university, a teaching-research university, a teaching university, or an application-oriented university, if you want to introduce academic professionals from other colleges and universities into the university, you should make up for the shortcomings and improve the level of disadvantaged resources according to the strength difference. Attracting the influx of academic professionals from the same type of university requires strengthening the resources of high-intensity factors affecting the flow of academic professionals and strengthening the level of superior resources. This requires managers to act in accordance with the law of flow on the basis of exploring the law of flow.

\section{Conclusions}

By analyzing the differences in the intensities of factors affecting the mobility of academic professionals in colleges and universities, we gain insight into the dynamics of the mobility of academic professionals and the theory of academic professionals' mobility is further enriched. In previous studies, scholars focused on the analysis of the state and types of influencing factors of the mobility of academic professionals in colleges and universities, but few people have conducted empirical analysis and comparison of the influencing factors of the mobility of academic professionals in different types of colleges and universities. For colleges and universities, the establishment of a team of academic professionals with optimized structure and high quality is the key to improving the level of colleges and universities, enhancing the overall strength of colleges and universities, and entering the ranks of "double first-class." In the process of development, colleges and universities have unscrupulously introduced high-level academic professionals in order to recruit high-quality student resources. Through the study of the influencing factors of the mobility of academic professionals in the academic labor market, the types of colleges are divided, the model of academic professionals mobility factors is established, and the policy recommendations to promote the mobility of academic professionals are provided to universities, which is conducive to helping colleges and universities to understand correctly. Factors affecting the mobility of academic professionals promote the mobility of academic professionals in colleges and universities. It can also positively guide the mobility of academic professionals in colleges and universities, regulate mobility behaviors, make the academic labor market more perfect, operate more effectively, and avoid vicious competition. The shortcomings of this study are due to the high difficulty of stratified sampling; this article adopts a combination of online survey and paper questionnaire survey, making the questionnaire stratified sampling fail to reach the initial design of the survey, and some colleges have fewer samples. The survey data between universities is not balanced, but fortunately, the collected samples meet the statistical requirements. Although not good enough, it does not affect the final statistical analysis. Secondly, the statistical analysis method of data is relatively simple, lacking in-depth research on the correlation of related factors or causality.

\section{Data Availability}

No data were used to support this study.

\section{Conflicts of Interest}

The author declares no conflicts of interest.

\section{Acknowledgments}

This study was supported by the Education and Teaching Reform Project of Heilongjiang Province (SJGY20180422 and SJGY20200602).

\section{References}

[1] Y. Zhou, Y. Guo, and Y. Liu, "High-level talent flow and its influence on regional unbalanced development in China," Applied Geography, vol. 91, pp. 89-98, 2018.

[2] A. Joseph, P. Rutz, S. Stachowiak, and S. Jaume, "Higher education analytics," International Journal of Information Systems and Social Change, vol. 8, no. 1, pp. 58-70, 2017.

[3] S. L. Zhao, D. Y. Zhu, X. B. Peng, and W. Song, "An empirical analysis of the regional competitiveness based on S\&T talents flow," Human Systems Management, vol. 35, no. 1, pp. 1-10, 2016.

[4] P. Finch, "Awards and competitions showthattalent will out," The Architects' Journal, vol. 244, no. 13, 16 pages, 2017.

[5] B. Q. Cai and X. H. Huang, "Evaluating the coordinated development of regional innovation ecosystem in China," Ekoloji, vol. 27, no. 106, pp. 1123-1132, 2018.

[6] B. Leavy, "Customer-centered innovation: improving the odds for success," Strategy \& Leadership, vol. 45, no. 2, pp. 3-11, 2017.

[7] L. Maxwell, "Superbosses: how exceptional leaders master the flow of talent," Library Journal, vol. 141, no. 2, 84 pages, 2016.

[8] G. William and O. Superbosses, "How exceptional leaders master the flow of talent, by sydney finkelstein," Organization Management Journal, vol. 13, no. 4, pp. 230-232, 2016. 
[9] M. J. E. Superbosses, "How exceptional leaders master the flow of talent," Journal of Applied Management and Entrepreneurship, vol. 21, no. 3, pp. 124-127, 2016.

[10] D. Hopkins, J. Higham, S. Tapp, and T. Duncan, "Academic mobility in the Anthropocene era: a comparative study of university policy at three New Zealand institutions," Journal of Sustainable Tourism, vol. 24, no. 3, pp. 376-397, 2016.

[11] M. Paula, "Vulnerability, gender and resistance in transnational academic mobility," Tertiary Education and Management, vol. 24, no. 3, pp. 254-265, 2018.

[12] X. Hao, G. Zhang, and S. Ma, “Deep learning," International Journal of Semantic Computing, vol. 10, no. 3, pp. 417-439, 2016.

[13] G. Litjens, T. Kooi, B. E. Bejnordi et al., "A survey on deep learning in medical image analysis," Medical Image Analysis, vol. 42 , no. 9, pp. 60-88, 2017.

[14] D. S. Kermany, M. Goldbaum, W. Cai et al., "Identifying medical diagnoses and treatable diseases by image-based deep learning," Cell, vol. 172, no. 5, pp. 1122-1131, 2018.

[15] D. Shen, G. Wu, and H.-I. Suk, "Deep learning in medical image analysis," Annual Review of Biomedical Engineering, vol. 19, no. 1, pp. 221-248, 2017

[16] Y. Chen, Z. Lin, Z. Xing et al., "Deep learning-based classification of hyperspectral data," IEEE Journal of Selected Topics in Applied Earth Observations \& Remote Sensing, vol. 7, no. 6, pp. 2094-2107, 2017.

[17] X. Wang, L. Gao, S. Mao et al., "CSI-based fingerprinting for indoor localization: a deep learning approach," IEEE Transactions on Vehicular Technology, vol. 66, no. 1, pp. 763-776, 2016.

[18] S. Albarqouni, C. Baur, F. Achilles, V. Belagiannis, S. Demirci, and N. Navab, "AggNet: deep learning from crowds for mitosis detection in breast cancer histology images," IEEE Transactions on Medical Imaging, vol. 35, no. 5, pp. 1313-1321, 2016.

[19] D. Ravi, C. Wong, F. Deligianni et al., "Deep learning for health informatics," IEEE Journal of Biomedical and Health Informatics, vol. 21, no. 1, pp. 4-21, 2017.

[20] D. Marmanis, M. Datcu, T. Esch, and U. Stilla, "Deep learning earth observation classification using ImageNet pretrained networks," IEEE Geoscience and Remote Sensing Letters, vol. 13, no. 1, pp. 105-109, 2016.

[21] G. Li, Y. Zhang, T. Deng et al., "Responses of tree stem sap flow and its main influencing factors to bud pruning," Nongye Gongcheng Xuebao/Transactions of the Chinese Society of Agricultural Engineering, vol. 37, no. 5, pp. 131-139, 2021.

[22] Y. Z. Hou, P. Li, J. Zhang et al., "Identification of critical influencing factors in dropping process of Xuesaitong Dropping Pills," Zhongguo Zhong yao za zhi=Zhongguo zhongyao zazhi=China journal of Chinese materia medica, vol. 46, no. 1, pp. 103-109, 2021.

[23] F. Xiong, L. Gan, and W. Sun, "Characterization of reservoir permeability and analysis of influencing factors in fracturepore medium," Chinese Journal of Geophysics-Chinese Edition, vol. 61, no. 1, pp. 279-288, 2021.

[24] P. Singh and R. Agrawal, "A customer centric best connected channel model for heterogeneous and IOT networks," Journal of Organizational and End User Computing, vol. 30, no. 4, pp. 32-50, 2018.

[25] S. Ivanaj, G.-B. Nganmini, and A. Antoine, "Measuring E-learners' perceptions of service quality," Journal of Organizational and End User Computing, vol. 31, no. 2, pp. 83-104, 2019.
[26] C.-L. Wei and C.-T. Ho, "Exploring signaling roles of service providers' reputation and competence in influencing perceptions of service quality and outsourcing intentions," Journal of Organizational and End User Computing, vol. 31, no. 1, pp. 86-109, 2019. 